\title{
Relación parental, autoestima y sintomatología depresiva en jóvenes adultos. Implicaciones de los conflictos interparentales, coalición y triangulación*
}

\section{Parental relationships, self-esteem and depressive symptoms in young adults. Implications of interparental conflicts, coalition and triangulation}

Recibido: julio 22 de 2012 | Revisado: mayo 26 de 2013 | Aceptado: enero 14 de 2014

\author{
CATARina Pinheiro Mota** \\ Universidade de Trás-os_Montes e Alto Douro, \\ Vila Real, Portugal \\ Paula Mena Matos \\ Universidad do Porto, Portugal
}

doi:10.11144/Javeriana.UPSY13-3.rpas

Para citar este artículo: Mota, C. P., \& Matos, P. M. (2014). Relación parental, autoestima y sintomatología depresiva en jóvenes adultos. Implicaciones de los conflictos interparentales, coalición y triangulación. Universitas Psychologica, 13(3), 907-922. http:/ dx.doi.org/10.11144/Javeriana.UPSY13-3.rpas

" Artículo de investigación. Este estudio se incluye en el Proyecto de investigación internacional - YAGISSP (Young Adults in Germany, Italy, Sweden, Spain and Portugal), que analiza la situación de adultos emergentes en Europa teniendo en cuenta una perspectiva intercultural. Este trabajo ha sido financiado por los Fondos FEDER a través del Programa Operacional de Factores de Competitividad - COMPETE y por Fondos Nacionales a través da FCT - Fundación para la Ciencia y la Tecnología en el ámbito del proyecto FCOMP-01-0124-FEDER-022714.

** Profesora Auxiliar de la Universidad de Trás-osMontes y Alto Douro - Departamento de Educación y Psicologia - Edifício do Complexo Pedagógico - UTAD, 5000-801, Vila Real. Portugal. catppmota@utad.pt. Investigadora en el Centro de Psicología de la Universidad do Porto. Portugal. Rua do Dr. Manuel Pereira da Silva, 4200-392 Porto, Portugal. telf. +351226079700; Fax. +351 226079 725.

**** Profesora Auxiliar de la Facultad de Psicología y de Ciencias de la Educación de la Universidad do Porto - FPCEUP. Investigadora en el Centro de Psicología de la Universidad do Porto. Rua do Dr. Manuel Pereira da Silva, 4200-392 Porto, Portugal. telf. $+351226079700 ;$ Fax. +351226079725.

\section{RES U MEN}

El presente estudio se propone examinar la asociación entre la calidad de la relación con los padres, la autoestima y la sintomatología depresiva, en una muestra de 584 jóvenes portugueses entre 18 y 30 años de edad. También se evalúa el papel mediador de los conflictos, en la asociación entre la calidad de la relación con los padres y la autoestima. La evaluación se ha hecho mediante los instrumentos Network Relationship Inventory, Children's Perception of Interparental Conflict Scale, Loyalty Conflict Scale, Self-Esteem Scale y Depression Scale. Los resultados determinan que la calidad de la relación con ambos padres disminuye el efecto del conflicto interparental, tal como de la coalición y la triangulación a los hijos. Para la madre, los conflictos ejercen un papel mediador entre la calidad de la relación con la madre y la autoestima. Los conflictos ejercen también un efecto negativo en la sintomatología depresiva. En el padre, se verifica una mediación de la autoestima por parte de la calidad de la relación con el padre y la sintomatología depresiva. El estudio destaca la necesidad de intervención en las competencias de los padres, para prevenir el desarrollo psicopatológico en los jóvenes.

Palabras clave

relación parental; autoestima; sintomatología depresiva; conflictos interparentales; jóvenes adultos.

\section{A B S T R A C T}

The present study examines the associations between quality of relationships with parents, self-esteem and depressive symptomatology in a sample of 584 Portuguese young adults, 18 to 30 aged. In addition, it tests the mediating role of interparental conflict in the association between quality of the relationship with parents and selfesteem. Evaluation was done through the Network Relationship Inventory, the Children's Perception of Interparental Conflict Scale, Loyalty Conflict Scale, the Self-Esteem Scale and the Depression Scale. The results indicated that the quality of the relationship with both parents decreases the effect of interparental conflict such as coalition and triangulation in young adults. Interparental conflict plays a mediating role between the quality of mother relationship and self-esteem. The conflicts have a negative effect on depression. Regarding the father relationship, self-esteem plays a meditating role in the association between quality of father relationship and depressive symptomatology. The study emphasizes intervention in parenting competencies to prevent psychopathology in youth development. Keywords

parental relationship; self-esteem; depressive symptomatology; interparental conflicts; young adult 


\section{Introducción}

La asociación entre la calidad del apego a los padres y el desarrollo de la salud mental en los jóvenes ha ganado recientemente un mayor impacto a través de un cuerpo robusto de investigaciones (e. g., Bifulco, Moran, Jacobs \& Bunn, 2009; Chung, Chen, Greenberger \& Heckhausen, 2009; Ngain, 2009; Parker \& Benson, 2004; Shaw \& Dallos, 2005). Acompañada por rápidos cambios físicos, psicológicos y sociales, la adolescencia y joven adultez representa una etapa del desarrollo particularmente caracterizada por la vulnerabilidad. La depresión es una de las principales causas de morbilidad en adolescentes y adultos jóvenes, tomando a menudo un curso crónico, recurrente o episódico (Merry, S., McDowell, H., Hetrick, S., Bir, J. \& Muller, N. (2004).

Algunos de los resultados negativos asociados con la depresión en adolescentes incluyen rendimiento académico, la disfunción familiar y social, semiología diversa como la anhedonía, labilidad emocional, sentimientos de ineficacia, falta de sueño y de apetito, intentos de suicidio y suicidio consumado (e. g., Beck, 1976; McGorry, Purcell, Hickie \& Jorm, 2007; Merry et al., 2004). Estas dificultades también son a menudo evidentes en los niños y adolescentes con niveles subclínicos de depresión (Gillham, Shatté \& Reivich, 2001), y pueden continuar en la edad adulta, donde pueden surgir nuevas dificultades laborales, económicas e interpersonales (Bayer \& Sanson, 2003; Shaw \& Dallos, 2005). Dada la alta tasa de prevalencia y los efectos negativos asociados, la necesidad de prevenir el desarrollo de trastornos depresivos se ha convertido en esencial. Una forma de lograr este objetivo ha sido la implementación de programas de prevención y el trabajo con los padres. Las dificultades en las relaciones interpersonales, así como la confrontación con situaciones adversas en el contexto familiar y social son factores de riesgo para el desarrollo de síntomas depresivos (e. g. Chango, McElhaney \& Allen, 2009).

La autoestima se define como el juicio las personas tienen con respecto a sí mismas (Rosenberg, 1979), mostrándose relacionada con la depresión (e. g., Cheng \& Furnham, 2003). Sin embargo, la autoestima es a menudo vista como un antecedente de depresión más que una consecuencia (e. g., Boden, Fergusson \& Horwood, 2008; Southall \& Roberts, 2002). Desde una perspectiva evolutiva, la autoestima está ligada a las experiencias sociales y desarrollo social temprano en el curso de la vida (Mruk, 2006). Así, los retrasos en el desarrollo de habilidades interpersonales y la necesidad de ser aprobado por los demás a menudo se asocian con la depresión (Harter, 1999, 2003).

El presente estudio se basa en la teoría del apego de Bowlby (1969/1982), cuyo abordaje traduce inherentemente la importancia de la calidad de la relación con los padres. Así mismo, las representaciones del autoconcepto, creadas tempranamente a través de la experiencia de apego, parecen desempeñar un rol importante en el desarrollo de una estructura de adaptación o de depresión a lo largo del ciclo vital.

Para los jóvenes, el sentido de pertenencia y de apoyo emocional de las figuras primarias de afecto es una necesidad constante. Sin embargo, aunque se verifique una disminución de la demanda física en la adolescencia y adultez, la proximidad emocional es un factor de seguridad capaz de garantizar su bienestar psicológico (e. g., Holmes, 2001; Wilkinson \& Watford, 2001). El presente estudio va de encuentro a esta idea, ya que los adolescentes y jóvenes adultos parecen beneficiarse del aumento de la autoestima y disminución de síntomas depresivos, durante el mantenimiento de relaciones de intimidad, afecto y de satisfacción con los padres.

Según algunos autores, el apoyo de los padres en virtud del bienestar de los jóvenes tiene un efecto positivo en los niveles de autoestima, que además parecen interferir en la reducción de síntomas depresivos (e. g., Elkington, Bauermeister \& Zimmerman, 2011; Parker \& Benson, 2004). A su vez, el desarrollo de una imagen positiva de sí mismos como elemento digno de afecto y aceptación de los padres parece reflejar un apego seguro que conduce a una mayor autoestima y menores niveles de depresión (e. g., Irons \& Gilbert, 2005; Shaw \& Dallos, 2005). Este resultado ha sido corroborado a través de estudios realizados con adolescentes y 
jóvenes adultos portugueses. Según estos estudios, los vínculos con las figuras parentales predicen significativamente el desarrollo de la autoestima (Mota \& Matos, 2009; Rocha, 2008) y disminuye el desarrollo de síntomas depresivos (Machado, Fonseca \& Queiroz, 2008).

Recientemente Buehler y Welsh (2009) sostuvieron que dificultades emocionales de los jóvenes debidas a la exposición continuada a conflictos interparentales, parece disminuir la seguridad interna y aumentar la vulnerabilidad a la psicopatología asociada, sobre todo a los síntomas depresivos. Así, se reconoce que a lo largo de la trayectoria de vida hay algunos factores de riesgo que podrían comprometer el bienestar de los jóvenes. Los conflictos interparentales son una variable relevante y sugieren una menor satisfacción personal y baja autoestima para los jóvenes; acentuándose siempre que existe la percepción de alta intensidad y frecuencia de los conflictos, a la vez de una baja resolución de los mismos (e. g., Buehler \& Welsh, 2009; Mota \& Matos, 2009).

Se señala que la permanencia del conflicto interparental parece afectar a los adolescentes y también a los jóvenes adultos, aunque de diferentes formas, originando sentimientos de culpa y de división, que en algunos casos sugiere la necesidad de encontrar seguridad a través del establecimiento de preferencias en la relación con los padres.

Los conflictos interparentales son uno de los aspectos que se destaca en este estudio, y que a su vez podrían impulsar otras variables igualmente importantes como los conflictos de lealtad -la triangulación y la coalición- en la relación con las figuras parentales. Además de los conflictos interparentales, es frecuente que los jóvenes tengan que atenerse a un proceso inherente de triangulación, entendido como "atrapado en el medio" (caught in the middle), representando muchas veces un vehículo de mensaje entre los padres (Minuchin, 1974). A su vez, este proceso sugiere que los jóvenes sean conducidos a una división personal entre los padres, lo que puede resultar en coalición. Este concepto, también abordado en el presente estudio, es más frecuente en adolescentes y también en jóvenes adultos, y representa el proceso de alianza con apenas una de las figuras parentales contra la otra (Buchanan \& Heiges, 2001).

Recientemente, Mota y Matos (2010) destacan la idea de que uno de los mayores protectores de los conflictos de lealtad - coalición y triangulación, es la calidad de la relación con los padres. Algunos estudios han señalado el efecto de la triangulación y de la coalición en el bienestar de los jóvenes, poniendo en relieve la baja autoestima, problemas de conducta, depresión y ansiedad (e. g., Buchanan \& Heiges, 2001; Franck \& Buehler, 2007; Gerard, Buehler, Franck \& Anderson, 2005). En este sentido, la relación de los hijos hacia el padre y la madre puede variar en lo que concierne a la forma de gestionar los conflictos. Pese a que la gran mayoría de estudios se centran en la relación con la madre, es sabido que la relación con las dos figuras parentales es distinta. Asimismo, aunque la madre pase habitualmente más tiempo con los hijos, es importante considerar los efectos de las dinámicas parentales de ambos (Hoeve, Dubas, Gerris, van der Laan \& Smeenk, 2011). Aparte de la cantidad de tiempo que cada uno permanece con los hijos, existen indicadores de que la implicación parental es cualitativamente distinta (Videon, 2005). De este modo, los padres habitualmente aportan niveles de cuidado instrumental más significativo que las madres, a la vez que estas asumen un mayor rol en los cuidados y en la implicación emocional (Williams \& Kelly, 2005). Por todo ello, en el presente estudio se aborda de forma distinta el análisis del padre y de la madre, ya que el componente emocional implicado en la calidad de la relación con las dos figuras parentales, en la autoestima y sintomatología depresiva posiblemente son distintos.

De hecho, aunque existen bastantes estudios centrados en el conflicto interparental, son escasos los estudios que abordan otros conceptos de conflictos en la relación padres-hijos como la triangulación y la coalición, sobretodo en jóvenes adultos. Por este motivo, este tema es de gran relevancia para nuevas investigaciones. Se destaca también el rol de la calidad de la relación con las figuras parentales, 
discutida bajo la teoría del apego, concepción teórica también presente en este trabajo. Igualmente, este estudio analiza la importancia de la calidad de la relación con los padres y su papel en los conflictos interparentales, los conflictos padres-hijos, la autoestima y la sintomatología psicopatológica de los jóvenes.

\section{Objetivos}

Por todo lo expuesto y dada la relevancia de la calidad de las relaciones emocionales, en el presente estudio se espera la asociación y el efecto predictivo de la calidad de la relación con los padres (incluyendo la intimidad, el afecto y la satisfacción) en la autoestima, así como en el desarrollo de sintomatología depresiva. A su vez, se espera un efecto de los conflictos interparentales, de la coalición y de la triangulación en la autoestima y el desarrollo de sintomatología depresiva en jóvenes. Por último, se espera el papel mediador de los conflictos en la asociación entre la calidad de la relación con los padres y la autoestima. Este análisis de los efectos de los conflictos será evaluado de forma separada para el padre y la madre (véase el modelo conceptual representado en la Figura 1).

\section{Método}

\section{Participantes}

La muestra está constituida por 584 jóvenes con edades entre 18 y los 30 años $\left(M_{\text {edad }}=21.08 ; \mathrm{DE}=\right.$ 2.88), de los cuales 228 (39\%) hombres y 356 (61\%) mujeres; 561 (96.1\%) solteros y 404 (69.2\%) viven con ambos padres, 56 (9.6\%) provienen de familias monoparentales -madre (divorciada o soltera) y solo 7 (1.2\%) de familias monoparentales -padre (divorciado); los restantes 20\% viven con otros familiares, solos o con amigos. Se destaca también que 457 (78.3\%) jóvenes frecuentan la universidad pública en su ciudad/país de origen y, de estos, solo 52 (8.9\%) se han desplazado de su ciudad de origen para estudiar.

\section{Instrumentos}

Network Relationship Inventory (Furman $\mathcal{E}$ Buhrmester, 1985)

Originalmente, consta de 28 ítems divididos en ocho dimensiones. En este estudio, solo se utilizaron las tres dimensiones (en un total de 11 ítems) que señalan los aspectos positivos de la calidad de las

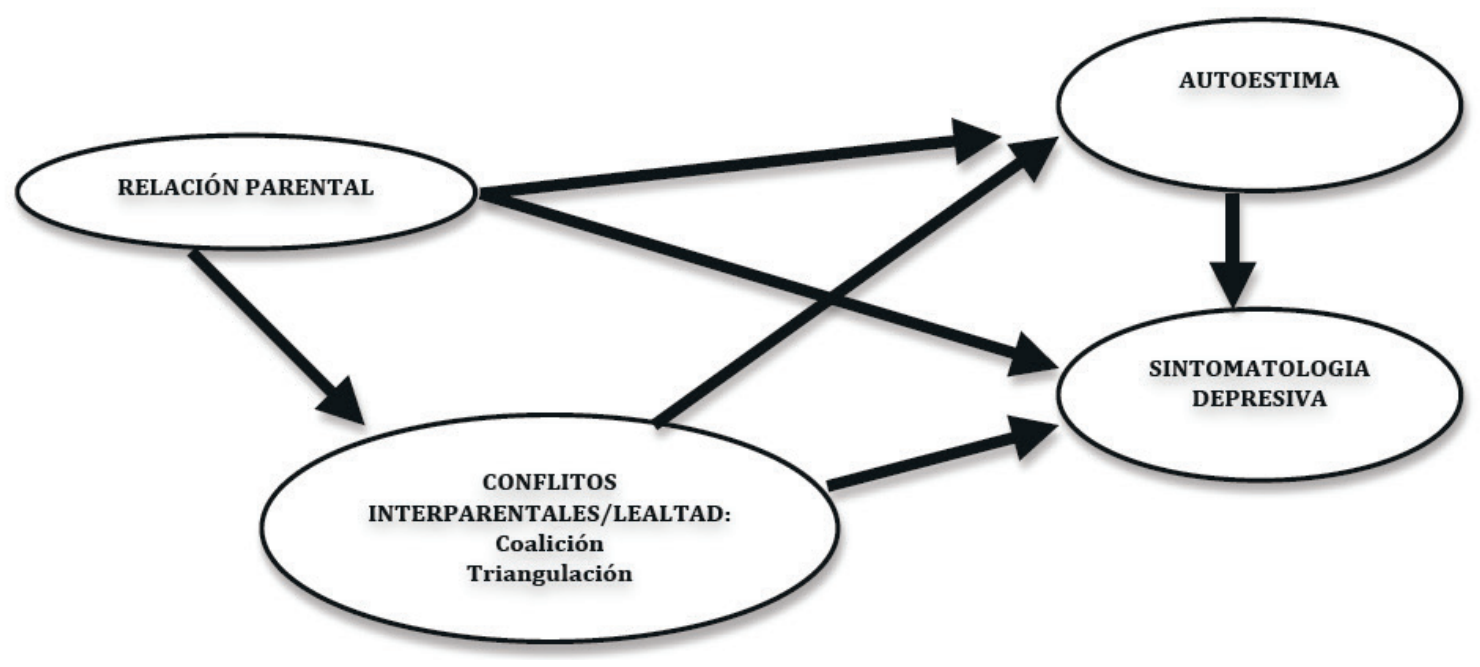

Figura 1. Modelo conceptual del estudio.

Fuente: elaboración propia 
relaciones con las figuras parentales: la Intimidad (3 ítems; por ejemplo: "Habla con sus padres de cosas que no quiere que otros tengan conocimiento"), la Satisfacción (2 ítems; "EEstá satisfecho en la relación que tiene con sus padres?") y el Afecto en su forma activa y pasiva ( 6 ítems; activa: "Siente amor y afecto por sus padres"; pasiva: "Es amado por sus padres"). Cada ítem utiliza una escala Likert con cinco alternativas de respuesta. La consistencia interna de los autores originales tiene un promedio de 0.8 (Furman \& Buhrmester, 1985). En Portugal ha sido usado en algunos estudios con alfas comprendidos entre 0.76 y 0.96 (Mota \& Matos, 2011b). En el presente estudio, la consistencia interna fue medida por alfa de Cronbach con los valores adecuados para la madre y el padre, respectivamente: Intimidad, $\alpha$ $=0.89 / 0.86$; Satisfacción, $\alpha=0.76 / 0.79$; Afecto, $\alpha=0.94 / 0.96$. Las propriedades psicométricas no se han alterado por el uso parcial del instrumento. Se realizó un análisis factorial confirmatorio de primer orden con todas las tres dimensiones del instrumento y se obtuvieron los valores de los índices principales de ajustes adecuados, para padre y madre, respectivamente $\left(\chi^{2}=1010.5_{(25)} ; p=0.000^{1}\right.$; $\mathrm{CFI}=0.94 ; \mathrm{RMSEA}=0.06 ; \chi^{2}=1373_{(27)} ; p=0$; $\mathrm{CFI}=0.91 ; \mathrm{RMSEA}=0.07$ ).

\section{Children's Perception of Interparental Conflict}

Scale (Grych, Seid Eु Fincham, 1992)

Es un instrumento con 49 items y con nueve dimensiones. En este estudio, se utilizaron dos dimensiones: la Frecuencia (3 ítems: "Mis padres discuten") y Resolución (1 ítem "Después de una discusión, mis padres se reconcilian"), confirmando un total de cuatro ítems. Cada ítem utiliza una escala Likert con cinco alternativas de respuesta. La consistencia interna de los autores originales varía entre 0.63 y 0.83 (Grych et al., 1992). En Portugal, se ha usado en varios estudios con alfas comprendidos entre 0.82 y 0.86 (e. g., Mota \& Matos, 2009; Moura,

1 Se observa significancia en el ji cuadrado contrariamente a lo que se esperaría, hecho que se relaciona con el reducido tamaño de la muestra. Lo mismo se constata en las demás ecuaciones a lo largo del estudio.
Andrade, Rocha \& Matos, 2010; Santos \& Matos, 2007). En el presente estudio, las dimensiones del instrumento utilizado muestran una consistencia interna con el alfa de Cronbach de 0.7. Las propriedades psicométricas no se han alterado por el uso parcial del instrumento.

Loyalty Conflict Scale (Wendt, Kroll, Beckh, Gerhard EO Walper 2002)

Se trata de una escala con 12 ítems divididos en dos dimensiones: la Coalición (6 ítems: "A mi madre le gustaría que yo la quisiera a ella más que a mi padre") y la Triangulación (6 ítems: "Me siento dividido entre mis padres"), con una escala de respuesta tipo Likert de cinco opciones. La consistencia interna de los autores originales varía entre 0.67 y 0.8 (Walper, Kruse, Noack \& Schwarz, 2005). El presente instrumento ha sido poco usado en Portugal, por lo que no se conocen estudios reportados con la consistencia interna. En el presente estudio la consistencia interna fue medida por el alfa de Cronbach, obteniéndose valores igualmente adecuados: Coalición: $\alpha=$ 0.8; Triangulación: $\alpha=0.85$. Se realizó un análisis factorial confirmatorio de primer orden con las dos dimensiones del instrumento, obteniéndose valores de los índices principales de ajustes adecuados $\left(\chi^{2}=222.4_{(50)} ; p=0\right.$; CFI $=0.95$; RMSEA $=0.08)$.

\section{Rosenberg's Self-Esteem Scale (Rosenberg, 1965)}

Escala de una sola dimensión que consta de 10 ítems destinados a la evaluación de la autoestima en los adolescentes ("Siento que tengo buenas cualidades"), mediante una escala tipo Likert de 6 puntos de evaluación cuantitativa. La consistencia interna de los autores originales varía entre 0.77 y 0.88 (Rosenberg, 1965). Este instrumento ha sido largamente utilizado en Portugal con una consistencia interna de 0.85 (e. g., Mota \& Matos, 2013; Rocha, Mota \& Matos, 2011). El alfa de Cronbach para esta muestra fue de 0.86 . El análisis factorial confirmatorio mostró adecuados índices de ajuste 
$\left(\chi^{2}=100.2_{(32)} ; p=0 ;\right.$ CFI $=0.96$, RMSEA $=$ 0.07).

\section{Depression Scale (Radloff, 1991)}

Se trata de una escala de una sola dimensión compuesta por ocho ítems destinados a evaluar el nivel de sintomatología depresiva en los jóvenes ("Durante esta semana me he sentido solo"), a través de una escala de respuesta tipo Likert de 5 puntos. Esta escala ha sido escasamente usada en Portugal por lo que se desconocen valores de consistencia interna. El alfa de Cronbach para esta muestra fue igualmente de 0.83 . El análisis factorial confirmatorio mostró adecuados índices de ajuste $\left(\chi^{2}=71.5_{(25)}\right.$; $p=0 ; \mathrm{CFI}=0.96$; $\mathrm{RMSEA}=0.07)$.

\section{Procedimiento}

Tratándose de un estudio transversal, la recogida de datos se realizó en un solo momento en las universidades y escuelas técnicas de la zona metropolitana de la ciudad de Porto (Portugal). La muestra fue recogida de forma aleatoria. La aplicación se llevó a cabo en horario escolar con el apoyo del equipo de investigación. Durante la administración, se presentaron los objetivos generales del estudio, por lo que, tratándose de una administración colectiva, se les dio instrucciones estándar de esclarecimiento en lo que respecta los cuestionarios de autoinforme, destacando en todo momento la confidencialidad de la información inherente a los cuestionarios y el carácter voluntario de la participación en el estudio. El consentimiento informado fue proporcionado por los jóvenes de forma oral accediendo a la participación en el estudio.

\section{Estrategia de análisis de datos}

Los datos fueron analizados de acuerdo con un modelo de ecuaciones estructurales: SEM (programa 6.1). El modelo muestra cómo las variables relacionales pueden influir entre sí y predecir los efectos en la autoestima y en la sintomatología depresiva en los jóvenes adultos. Se utilizó el método de máxima verosimilitud, habiendo sido anteriormente testada la normalidad de la muestra en estudio. Fueron verificadas las medidas de asimetría y curtosis que se encuentran dentro de los parámetros de - 1 y 1 , y la normalidad se corroboró por observación del ajuste de los gráficos Normal Q-Q Plots y Detrended Normal Q-Q Plots (Pallant, 2011; Larson-Hall, 2010).

Para la elaboración del modelo hemos recurrido al método de parceling o parcelamiento dado el elevado número de ítems, cuyos análisis desarrollan un elevado número de parámetros que se deben estimar en relación con el tamaño de la muestra. Esta metodología implica la agregación de los ítems y se relaciona con el aumento de la estabilidad de los parámetros que se deben valorar y la mejoría de la relación entre las variables y la dimensión de la muestra (Bandalos \& Finney, 2001).

En el análisis de acuerdo con el test de Sobel (Baron \& Kenny, 1986) se ha observado la existencia de efectos mediadores. El efecto mediador es evaluado de acuerdo con cuatro etapas principales: (a) la predicción significativa de la variable independiente (relación con los padres) sobre la variable dependiente (autoestima); (b) la predicción significativa de la variable independiente (relación con los padres) sobre la variable mediadora (conflictos), (c) y a su turno de la variable mediadora (conflicto) en la variable dependiente (autoestima) y por último, (d) la introducción de la variable mediadora (conflictos) en conjunto con la predicción de la variable independiente (relación con los padres) sobre la dependiente (autoestima), en que esta asociación debe bajar (mediación parcial) o desaparecer su asociación (mediación total). Analizando la descomposición de los efectos (por el valor de $\beta$ estandardizado, el error en su valor no estandardizado y la probabilidad con significancia de 0.5 ) se constató además de la existencia de la mediación la presencia de efectos indirectos (Preacher \& Hayes, 2004).

\section{Resultados}

\section{Resultados descriptivos}

Los análisis preliminares incluyen el cálculo de las medias y desviaciones típicas de las variables del estudio (Tabla 1). Los resultados observados muestran medias 
TABLA 1

Medias y desviación estándar de las variables en estudio

\begin{tabular}{lcc}
\hline \multicolumn{1}{c}{ Variables del estudio } & M & DE \\
\hline Conflictos & & \\
Conflictos Interparentales & 2.2 & 0.7 \\
Triangulación & 1.5 & 0.551 \\
Coalición & 1.6 & 0.735 \\
Autoestima & 3.2 & 0.516 \\
Sintomatología Depresiva & 1.7 & 0.549 \\
NRI Madre & & \\
Satisfacción & 4 & 1.02 \\
Intimidad & 2.7 & 1.17 \\
Afecto & 4.5 & 0.771 \\
NRI Padre & & \\
Satisfacción & 3.6 & 1.17 \\
Intimidad & 2.3 & 1.09 \\
Afecto & 4.3 & 0.978 \\
\hline
\end{tabular}

Fuente: elaboración propia

TABLA 2

Matriz de correlación entre las variables

\begin{tabular}{|c|c|c|c|c|c|c|c|c|}
\hline & \multicolumn{3}{|c|}{ Conflictos } & \multicolumn{3}{|c|}{ NRI } & \multirow[t]{2}{*}{ Autoestima } & \multirow{2}{*}{$\begin{array}{l}\text { Sintomat } \\
\text { Depresiva }\end{array}$} \\
\hline & Triangulación & Coalición & $\begin{array}{l}\text { Conf. Inter- } \\
\text { parentales }\end{array}$ & $\begin{array}{l}\text { Satisfacción } \\
\text { Madre/Padre }\end{array}$ & $\begin{array}{c}\text { Afecto } \\
\text { Madre/Padre }\end{array}$ & $\begin{array}{c}\text { Intimidad } \\
\text { Madre/Padre }\end{array}$ & & \\
\hline $\begin{array}{l}\text { Conflictos } \\
\text { Triangulación }\end{array}$ & 1 & $0.533^{* *}$ & $0.564 * *$ & $-0.258^{* * / 0} / 0.304^{* *}$ & $-0.176^{* * /-0.176^{* *}}$ & $-0.097 * /-0.201 * *$ & -0.169 ** & $0.225 * *$ \\
\hline Coalición & & 1 & $0.478^{* *}$ & $-0.395 * * /-0.422 * *$ & $-0.319 * * /-0.369 * *$ & $-0.195 * * /-0.284 * *$ & -0.147 & $0.179 * *$ \\
\hline Conf. Interparentales & & & 1 & $-0.225 /-0.35^{* *}$ & $-0.136 * * /-0.229 * *$ & $-0.064 /-0.176^{* *}$ & $-0.131 * *$ & $0.196 * *$ \\
\hline $\begin{array}{l}\text { NRI Satisfacción } \\
\text { Madre/Padre }\end{array}$ & & & & 1 & $\begin{array}{l}0.721^{* *} / 0.463^{* *} \\
0.462 * * / 0.735^{* *}\end{array}$ & $\begin{array}{l}0.613^{* *} / 0.451 * * \\
0.360^{* *} / 0.639 * *\end{array}$ & $\begin{array}{l}0.241 * * 1 \\
0.244^{* *}\end{array}$ & $\begin{array}{c}-0.211 * * /- \\
0.214^{* *}\end{array}$ \\
\hline $\begin{array}{l}\text { Afecto } \\
\text { Madre/Padre }\end{array}$ & & & & & 1 & $\begin{array}{l}0.465 * * / 0.304 * * \\
0.304 * * / 0.494 * *\end{array}$ & $\begin{array}{l}0.208 * * / \\
0.215^{* *}\end{array}$ & $\begin{array}{c}-0.183^{* *} /- \\
0.225^{* *}\end{array}$ \\
\hline $\begin{array}{l}\text { Intimidad } \\
\text { Madre/Padre }\end{array}$ & & & & & & 1 & $\begin{array}{c}0.123^{* *} / \\
0.16^{* *}\end{array}$ & $\begin{array}{l}-0.036 /- \\
0.122 * *\end{array}$ \\
\hline Autoestima & & & & & & & 1 & $-0.476^{* *}$ \\
\hline $\begin{array}{l}\text { Sintomatología } \\
\text { Depresiva }\end{array}$ & & & & & & & & 1 \\
\hline
\end{tabular}

$* p<0.05 . * * p<0.001$

Fuente: elaboración propia

relativamente bajas en los conflictos y sintomatología depresiva. Por otra parte, se verifican medias que oscilan entre niveles medios y medios-altos de la relación parental y la autoestima. Además, se ha determinado una matriz de correlación en el sentido de expresar las relaciones entre las variables del estudio (Tabla 2). Tal como se esperaba, las dimensiones del conflicto se correlacionaron negativamente con la calidad de la relación con la madre (aunque en menor intensidad) y con el padre, así como con la autoestima. Por otra parte, la calidad de la relación con la madre y el padre se correlacionaron positivamente con la autoestima y negativamente con la sintomatología depresiva. Es de destacar que, tal como se esperaba, la autoestima se encuentra negativamente correlacionada con la sintomatología depresiva. 


\section{Calidad de la relación con las figuras parentales}

En el modelo de ecuaciones estructurales construido para ambas figuras parentales, se ha decidido introducir el conflicto como variable mediadora de la asociación entre la calidad de la relación con los padres y la autoestima. Este análisis se basa en un sólido fundamento teórico que soporta la idea de que la calidad de la relación con la madre y el padre representa un factor protector para la adaptación psicosocial de los jóvenes (Bowlby, 1969/1982). Inclusive, tal como se ha hecho referencia a lo largo del estudio, también es cierto que algunos factores pueden dificultar esta tarea. Los conflictos interparentales conducen, frecuentemente, al desarrollo de conflictos de lealtad (coalición y triangulación), que a su vez condicionan la calidad de la relación con los padres e incluso afectan la autoestima de los jóvenes, desencadenando procesos depresivos.

\section{Calidad de la relación con la madre}

El análisis del efecto mediador llevó a cabo la elaboración de varios modelos; es así como la primera etapa comporta la predicción de la calidad de la relación con la madre hacia el conflicto (modelo 1a con $B=-0.22 ; p<0.01$ ), la autoestima (modelolb con $\beta=0.28 ; p<0.01)$ y la depresión (modelo 1c con $\beta=-0.24 ; p<0.01$ ); en el segundo paso (modelo 2 ) se observó la predicción de la autoestima por parte del conflicto $(B=-0.22 ; p$ $<0.01$ ); en el modelo 3 se presenta la predicción de la autoestima hacia la depresión $(B=-0.55 ; p$ $<0.01$ ) y la última etapa (modelo 4) está conformada por el modelo final en que el conflicto es introducido como mediador (Tabla 3). Tal como se observa en la Figura 2, los resultados permiten apreciar que la calidad de la relación con la madre (donde se incluye la satisfacción, la intimidad y el afecto) predice negativamente el conflicto $(\beta=$ $-0.38, p<0.01$ ), en concreto, el conflicto interparental además de la coalición y triangulación de padres-hijos.

Por otro lado, se observa que la autoestima es positivamente predicha por la calidad de relación a la madre $(\beta=0.25, p<0.01)$. La relación con la madre también parece ejercer un efecto negativo en el desarrollo de la sintomatología depresiva en los jóvenes (aunque no directo), por lo que constatamos un efecto indirecto y negativo a través de la autoestima $(B=0.13, p<0.01)$. Tal como se esperaba, el conflicto ejerce un efecto negativo en la autoestima $(B=-0.12, p<0.01)$. Los análisis mediacionales permitieron observar el efecto mediador (aunque parcial) de los conflictos entre la calidad de la relación con la madre y la autoestima $(z=2.17, E P=$ $0.008, p<0.05, \beta=0.05$ ). Cabe aún destacar el efecto indirecto positivo que el conflicto ejerce en la sintomatología depresiva a través de la autoestima ( $($ $=0.06, p<0.01)$. Por último, la autoestima predice negativamente el desarrollo de la sintomatología depresiva $(B=-0.52, p<0.01)$ (Figura 2$)$. El modelo de ecuaciones estructurales construido para la madre presentó índices de ajuste adecuados $\left(\chi^{2}=80.5_{(10)}\right.$; $p=0 ; \mathrm{CFI}=0.98 ; \mathrm{RMSEA}=0.05)$.

TABLA 3

Índices de ajuste de los modelos estimados para la madre

\begin{tabular}{clccccc}
\hline \multicolumn{1}{c}{ Modelo } & \multicolumn{1}{c}{ Descripción } & df & $\chi^{2}$ & RMSEA & CFI & $\beta$ \\
\hline Modelo 1a & Relación Madre - Conflicto & 25 & 147.33 & 0.09 & 0.92 & -0.22 \\
Modelo 1b & Relación Madre - Autoestima & 25 & 147.33 & 0.09 & 0.92 & 0.28 \\
Modelo 1c & Relación Madre - Depresión & 25 & 147.33 & 0.09 & 0.92 & -0.24 \\
Modelo 2 & Conflicto - Autoestima & 5 & 17.94 & 0.06 & 0.99 & -0.22 \\
Modelo 3 & Autoestima - Depresión & 7 & 24.69 & 0.03 & 0.99 & -0.55 \\
Modelo 4 & Modelo Mediador (Relación Madre -Conflicto-Autoestima) & 42 & 80.5 & 0.05 & 0.98 & 0.05 \\
\hline
\end{tabular}

Nota. $X^{2}=$ Chi-square; RMSEA = root mean square error of approximation; $\mathrm{CFI}=$ comparative fit index; $\beta=$ standardized value

Fuente: elaboración propia 


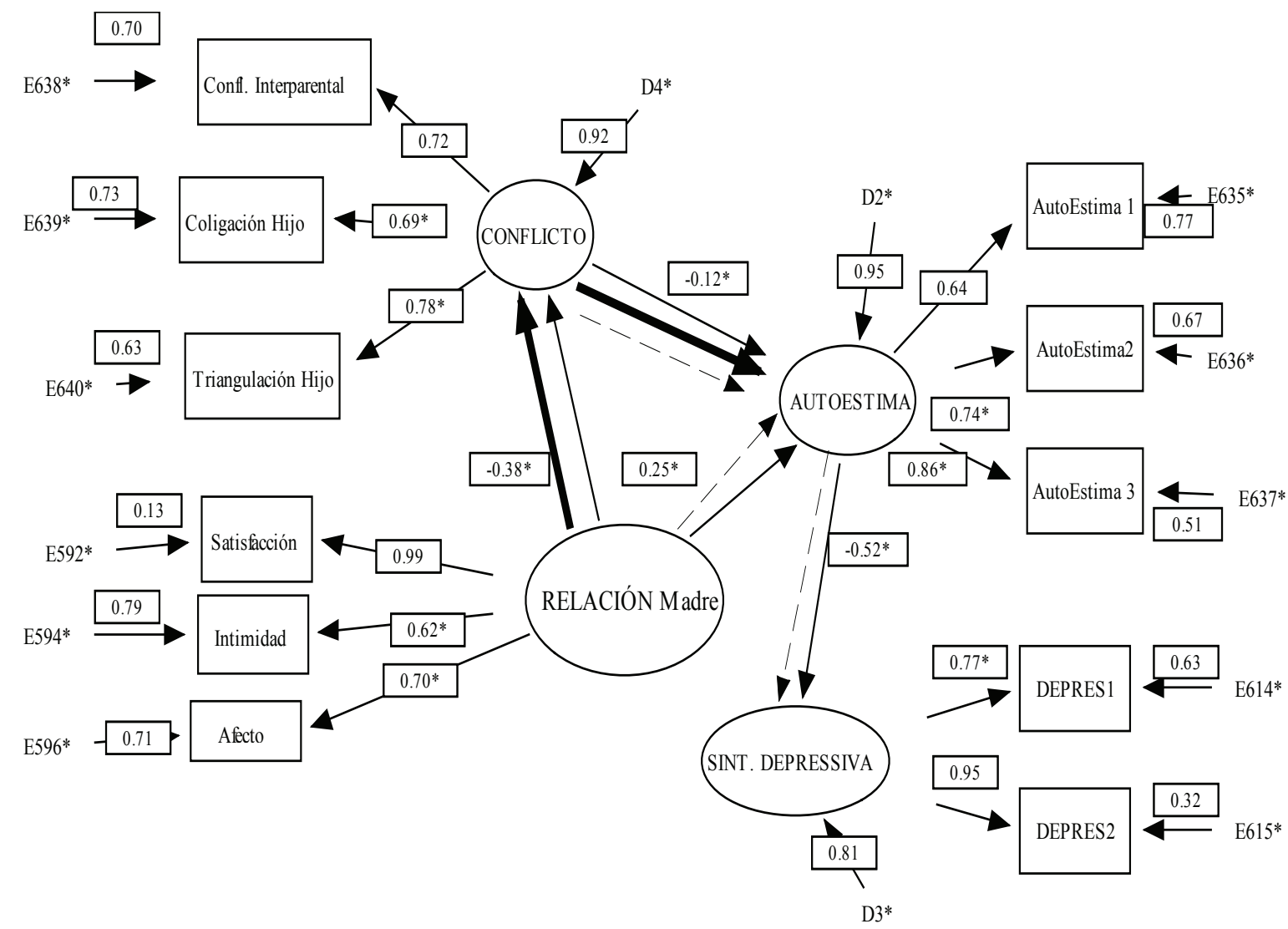

Efecto Indirecto

Efecto Mediador $(z=2.17, \mathrm{EP}=0.008, \mathrm{p}<0.05, \beta=0.05)\left(\chi^{2}=80.5_{(42)} ; \mathrm{p}=0 ; \mathrm{CFI}=0.98 ; \mathrm{RMSEA}=0.05\right)$

Figura 2. Modelo final representativo del efecto de la calidad de la relación con la madre en la autoestima, la sintomatología depresiva y los conflictos

Fuente: elaboración propia

\section{Calidad de la relación con el padre}

Tal como se ha desarrollado para la madre, el análisis del efecto mediador llevo a cabo la elaboración de varios modelos; es así como la primera etapa comporta la predicción de la calidad de la relación con el padre hacia el conflicto (modelo 1a con $B$ $=-0.51 ; p<0.01$ ), la autoestima (modelo $1 \mathrm{~b} \operatorname{con} \beta$ $=0.33 ; p<0.01$ ) y la depresión (modelo $1 \mathrm{c} c \operatorname{con} \beta$ $=-0.28 ; p<0.01$ ); en el segundo paso (modelo 2 ) se observó la predicción de la autoestima por parte del conflicto ( $(=-0.22 ; p<0.01$ ); en el modelo 3 se presenta la predicción de la autoestima hacia la depresión $(B=-0.58 ; p<0.01)$ y la última etapa (modelo 4) comporta el modelo final en que el conflicto es introducido como mediador (Tabla 4).
Tal como en el caso de la madre, el modelo final de ecuaciones estructurales presenta índices de ajuste adecuados $\left(\chi^{2}=88.5_{(42)} ; p=0 ; C F I=0.98\right.$; RMSEA $=0.05)$. La calidad de la relación con el padre (donde se incluye la satisfacción, la intimidad y el afecto) es la variable que más predice, de forma significativa y negativamente $(B=-0.51, p$ $<0.01$ ), el efecto del conflicto, a saber el conflicto interparental, además de la coalición y triangulación de padres-hijos, seguida de la autoestima que predice de forma positiva $(B=0.31, p<0.01)$. La relación con el padre también ejerce un efecto negativo $(B=-0.11, p<0.01)$ en el desarrollo de la sintomatología depresiva en los jóvenes. Por otra parte, se observó un efecto mediador negativo de la autoestima entre la calidad de la relación con el 
TABLA 4

Índices de ajuste de los modelos estimados para el padre

\begin{tabular}{clccccc}
\hline Modelo & \multicolumn{1}{c}{ Descripción } & $d f$ & $\chi^{2}$ & RMSEA & CFI & $\beta$ \\
\hline Modelo 1a & Relación Padre - Conflicto & 41 & 186.31 & 0.09 & 0.93 & -0.51 \\
Modelo 1b & Relación Padre - Autoestima & 41 & 186.31 & 0.09 & 0.93 & 0.33 \\
Modelo 1c & Relación Padre - Depresión & 41 & 186.31 & 0.09 & 0.93 & -0.28 \\
Modelo 2 & Conflicto - Autoestima & 8 & 8.2 & 0.01 & 0.99 & -0.22 \\
Modelo 3 & Autoestima - Depresión & 5 & 4.2 & 0.01 & 0.99 & -0.58 \\
Modelo 4 & Modelo Mediador (Relación Padre-Conflicto - Autoestima) & 42 & 88.5 & 0.05 & 0.98 & 0.16 \\
\hline
\end{tabular}

Nota. $X^{2}=$ Chi-square; RMSEA = root mean square error of approximation; $C F I=$ comparative fit index; $\beta=$ standardized value

Fuente: elaboración propia

padre y la depresión $(z=-4.43, E P=0.015, p=0$, $\beta=0.16)$. A su vez, la autoestima predice negativamente la sintomatología depresiva $(B=-0.53, p$ $<0.01$ ). Contrariamente a la relación con la madre, en el modelo de mediación, el conflicto no ejerce un efecto significativo en la autoestima $(\beta=-0.12$, $p>0.05$ ) (Figura 3).

\section{Discusión}

Los resultados del presente estudio corroboran la literatura más reciente que propone que la calidad de la relación con los padres constituye un factor con capacidad de predecir los efectos de los conflictos interparentales, de la coalición y de la triangulación, además del desarrollo de la psicopatología depresiva en los jóvenes (e. g., Buchanan \& Heiges, 2001; Franck \& Buehler, 2007; Gerard et al., 2005; Mota \& Matos, 2010). De acuerdo a las hipótesis propuestas obsérvese que la relación con ambos padres presenta un efecto negativo en los conflictos interparentales y conflictos padres-hijos. Al mismo tiempo, la calidad de la relación con los padres se correlaciona positivamente con el desarrollo de la autoestima, al tiempo que ejerce un efecto negativo en el desarrollo de la sintomatología depresiva. Sin embargo, la autoestima, corroborando la hipótesis propuesta, parece jugar un papel clave para predecir el desarrollo de sintomatología depresiva, presentando un valor robusto y negativo a este respecto. En ambas figuras parentales se observa que la calidad de la relación con los padres aumenta la autoestima y disminuye la sintomatología depresiva. En particular para el modelo del padre, la autoestima ejerce un efecto mediador parcial hacia la depresión. Por lo tanto, la calidad de la relación con los padres parece favorecer el desarrollo de un proceso de crecimiento personal, que incluye la autoestima como factor protector (Parker \& Benson, 2004). Así mismo niveles significativos de autoestima se asocian con jóvenes más seguros, aceptantes y realizados y menos propensos al desarrollo de enfermedades asociadas como la depresión (e. g., Bifulco et al., 2009; Shaw \& Dallos, 2005).

De este modo, los resultados de este estudio indican que las características positivas de la relación padres-hijos, como la intimidad, la satisfacción y el afecto hacia los padres pueden reflejar un alto grado de cercanía, participación y bienestar personal, lo que a su vez parece protegerlos frente a los factores de riesgo inherentes al contexto relacional, como la afectación de los conflictos y, de forma indirecta, la baja autoestima y la sintomatología depresiva.

Además de estos datos, en el presente estudio se destaca el conflicto interparental como un factor de riesgo, cuando la frecuencia y la intensidad no se acompañan de una solución constructiva capaz de permitir a los jóvenes un proceso de crecimiento personal. Confirmando la hipótesis de este trabajo, en particular se observa en la madre que los conflictos ejercen un efecto mediador negativo y parcial entre la relación con la madre y la autoestima. En esta medida, estudios con jóvenes de diferentes configuraciones familiares demuestran que la permanencia de conflictos interparentales y la falta de estrategias de resolución constructiva pueden acarrear conflictos en la relación con sus hijos (e. g., Mota \& Matos, 2011a). Las situaciones 


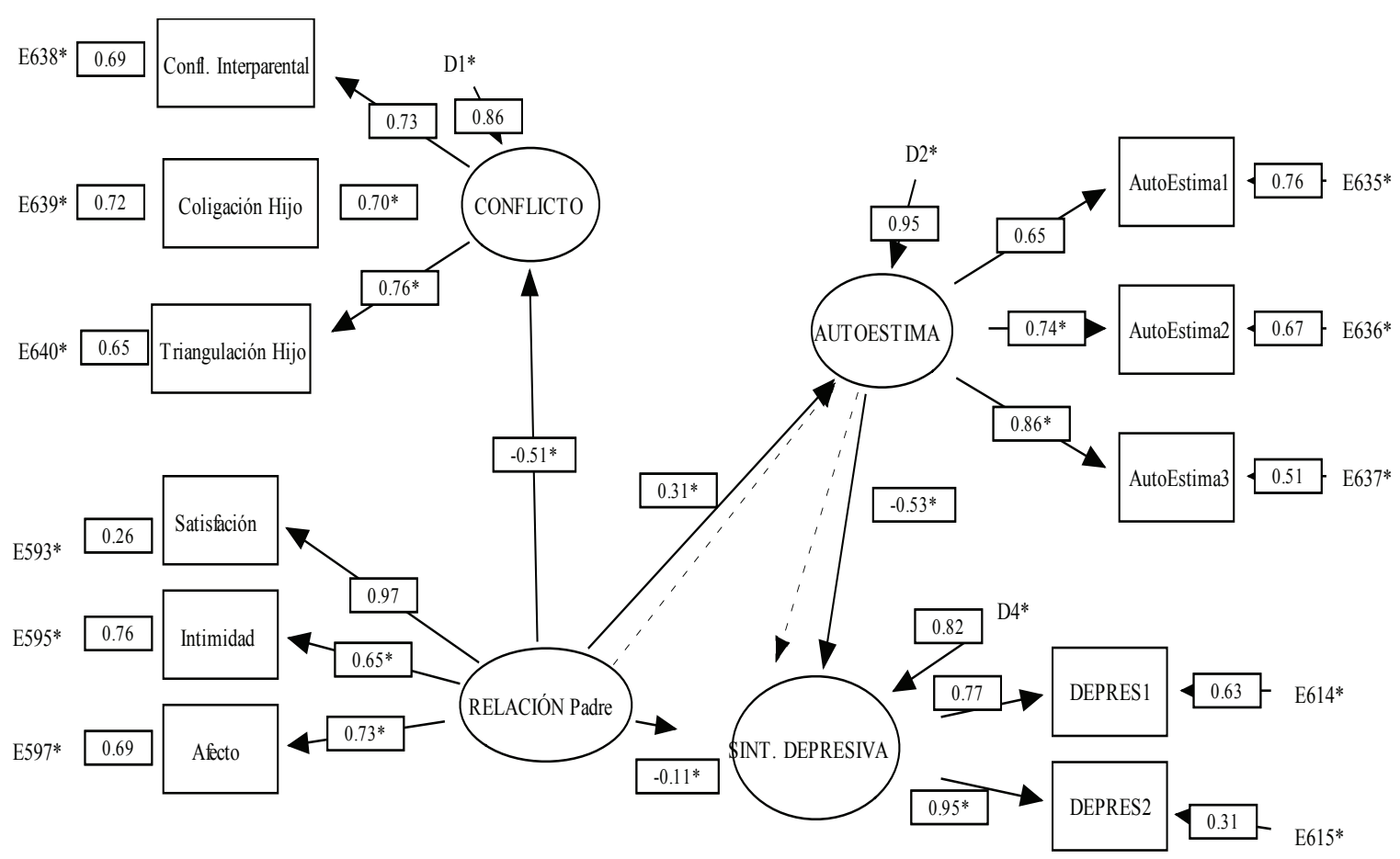

.......... Efecto Mediador $(z=-4.43, \mathrm{EP}=0.015, p=0, \beta=0.16)$

$\left(\chi^{2}=88.5_{(42)} ; p=0 ; \mathrm{CFI}=0.98 ; \operatorname{RMSEA}=0.05\right)$

Figura 3. Modelo final representativo del efecto de la calidad de la relación con el padre en la autoestima, la sintomatología depresiva y los conflictos

Fuente: elaboración propia

más comunes se refieren precisamente a los procesos de coalición.

Según Amato y Afifi (2006), la coalición es el resultado de un condicionamiento que los jóvenes sienten en relación con las opciones de los padres, de modo que la posición adoptada de mayor proximidad a uno de los padres, en la mayoría de los casos, conduce a sentimientos de angustia y culpa. A su vez, frecuentemente los resultados de la coalición crean un camino para que los jóvenes "se sientan atrapados en el medio" lo que se denomina de triangulación (e. g., Buchanan \& Waizenhofer, 2001; Walper \& Schwarz, 2001). El presente estudio apoya estas cuestiones, dado que la calidad de la relación con ambos padres parece disminuir el desarrollo de los efectos de los conflictos interparentales, así como la coalición y la triangulación en la relación.

Además de esta asociación y de acuerdo a la hipótesis delineada se percibe que los conflictos con la madre pueden implicar un efecto negativo significativo, aunque indirecto, en la predicción del desarrollo de los procesos depresivos. Así, como se comentó anteriormente, los resultados se relacionan con una mayor sensibilidad por parte de los jóvenes hacia la figura materna, que pueden ser afectados por una mayor sensibilidad y preocupación con el bienestar de la madre. En este contexto, autores como Buehler y Welsh (2009) añaden a esta perspectiva el hecho de que los conflictos de lealtad, como la triangulación, se asocian a la internalización de los problemas de los jóvenes y, como consecuencia, pueden producir sintomatología depresiva.

Sin embargo, aunque exista un factor de calidad relacional capaz de proteger los jóvenes del riesgo, se constataron algunas diferencias entre el padre y la madre, en cuanto al papel de los conflictos. Así, en el modelo que presenta la calidad de relación con la madre, los conflictos parecen jugar un papel importante en la predicción de la autoestima y también un papel mediador en la asociación entre la calidad de la relación con la 
madre y la autoestima. En cambio, lo mismo no se verifica con la figura del padre. Por lo tanto, se considera relevante entender este problema a raíz de la cercanía emocional que se desarrolla con la figura materna. De este modo, la calidad de la relación con la madre ejerce un papel protector de los conflictos (con un efecto positivo en la autoestima), por lo que los jóvenes parecen ser más sensibles a los conflictos y a las asociaciones con la figura materna (a través de la coalición y la triangulación), lo que puede disminuir la autoestima (Fosco \& Grych, 2008). En lo que concierne el modelo del padre, se identificó igualmente un efecto significativo y negativo de la relación con el padre en los conflictos. Al contrario de lo observado con la madre, estos no ejercen un papel mediador con la autoestima. Así mismo, podría decirse que la autoestima no depende de la forma como los jóvenes entienden los conflictos con el padre; no obstante, la calidad de la relación con el padre sí que resulta relevante para la salud mental de los jóvenes, una vez que la autoestima ejerce un rol mediador hacia el desarrollo de sintomatología depresiva. Por esta razón, jóvenes que revelan una autoestima significativa podrían estar más protegidos contra el desarrollo de sintomatología depresiva por sentirse más autoconfiados y seguros.

De este modo, discutiendo los resultados en una perspectiva más práctica, aunque en este estudio se considere apenas la perspectiva de los jóvenes, se destaca la necesidad de intervención y formación con jóvenes y padres, en el sentido de desarollar las competencias de las figuras parentales para prevenir el desarrollo psicopatológico en los jóvenes, sea por formación en la gestión de conflictos, educación parental o recurrencia a terapia familiar. Por lo tanto, padres más competentes en la gestión de los conflictos interparentales están también más disponibles emocionalmente. La resolución saludable de las disputas parentales, y sobretodo la forma como es entendida por los hijos, puede evitar el desarrollo de coalición y triangulación en la relación. Así mismo, padres seguros con una elevada proximidad hacia los hijos podrían potenciar la autoestima como factor protector de la sintomatología psicopatológica.

\section{Limitaciones del estudio}

Tratándose de un estudio transversal, la muestra fue recogida en un solo momento creando un compromiso a la discusión de los efectos causales y su dirección. Por otra parte, la muestra fue recogida en su mayoría en las universidades, con jóvenes estudiantes, en condiciones generales de vida en familias intactas, por lo que los resultados no representan una muestra aleatoria. Sería importante controlar algunas variables como por ejemplo la configuración familiar. Aunque no era un objetivo de este estudio, sería pertinente el análisis de la dinámica de los conflictos en otras configuraciones familiares mayoritarias (familias monoparentales o divorciados), o incluso tener en cuenta apenas una configuración familiar para distinguir los efectos. Del mismo modo, la recopilación de información se realizó a través de instrumentos de autoinforme, que en sí, son susceptibles de distorsión de la información, por lo que sería conveniente usar en futuros estudios investigaciones con otros informantes, incluyendo la perspectiva de los padres.

\section{Referencias}

Amato, P. R., \& Afifi, T. D. (2006). Feeling caught between parents: Adult children's relations with parents and subjective well-being. Journal of Marriage and Family, 68(1), 222-235.

Bandalos, D. L., \& Finney, S. J. (2001). Item parceling issues in structural equation modeling. En G. A. Marcoulides \& R. E. Schumacker (Eds.), Advanced structural equation modeling: New developments and techniques (pp. 269-296). Mahwah, NJ: Lawrence Erlbaum Associates, Inc.

Bayer, J. K., \& Sanson, A. V. (2003). Preventing the development of emotional mental health problems from early childhood: Recent advances in the field. International Journal of Mental Health Promotion, 5(3), 4-16.

Baron, R. M., \& Kenny, D. A. (1986). The moderatormediator variable distinction in social psychological research: Conceptual, strategic, and statistical considerations. Journal of Personality $\mathbb{E}$ Social Psychology, 51(6), 1173-1182. 
Beck, A. T. (1976). Cognitive therapy and emotional disorders. New York: International University Press.

Bifulco, A., Moran, P., Jacobs, C., \& Bunn, A. (2009). Problem partners and parenting: Exploring linkages with maternal insecure attachment style and adolescent offspring internalizing disorder. Attachment $\mathbb{E}$ Human Development, 11(1), 69-85.

Boden, J. M., Fergusson, D. M., \& Horwood, L. J. (2008). Does adolescent self-esteem predict later life outcomes? A test of the causal role of self-esteem. Development and Psychopathology, 20(1), 319-339.

Bowlby, J. (1969/1982). Attachment and loss: Vol. 1. Attachment (2.a ed.). New York: Basic Books.

Buchanan, C. M., \& Heiges, K. L. (2001). When conflict continues after the marriage ends: Effects of post-divorce conflict on children. En J. H. Grych $\&$ F. D. Fincham (Eds.), Interparental conflict and child development: Theory, research, and applications (pp. 337-362). Cambridge, U K: Cambridge University Press.

Buchanan, C. M., \& Waizenhofer, R. (2001). The impact of interparental conflict on adolescent children: Considerations of family systems and family structure. En A. Booth, A. C. Crouter \& M. Clements (Eds.), Couples in conflict (pp. 149-160). Mahwah, NJ: Erlbaum.

Buehler, C., \& Welsh, D. P. (2009). A process model of adolescent's triangulation into parent's marital conflict: The role of emotional reactivity. Journal of Family Psychology, 23(2), 167-180.

Chango, J. M., McElhaney, K. B., \& Allen, J. P. (2009). Attachment organization and patterns of conflicto resolution in friendships predicting adolescents' depressive symptoms over time. Attachment $\mathcal{E}^{2}$ Human Development, 11(4), 331-346.

Cheng, H., \& Furnham, A. (2003). Personality, selfesteem, and demographic predictions of happiness and depression. Personality and Individual Differences, 34(6), 921-942.

Chung, W. Y., Chen, C., Greenberger, E., \& Heckhausen, J. (2009). A cross-ethnic study of adolescents' depressed mood and the erosion of parental and peer warmth during the transition to young adulthood. Journal of Research on Adolescence, 19(3), 359-379.
Elkington, K. S., Bauermeister, J. A., \& Zimmerman, M. A. (2011). Do parents and peers matter? A prospective socio-ecological examination of substance use and sexual risk among African American youth. Journal of Adolescence, 34(5), 1035-1047.

Fosco, G. M., \& Grych, J. H. (2008). Emotional, cognitive, and family systems mediators of children's adjustment to interparental conflict. Journal of Family Psychology, 22(6), 843-854.

Franck, K. L., \& Buehler, C. (2007). A family process model of marital hostility, parental depressive affect, and early adolescent problem behavior: The roles of triangulation and parental warmth. Journal of Family Psychology, 21(4), 614-625.

Furman, W., \& Buhrmester, D. (1985). Children's perceptions of the personal relationships in their social networks. Developmental Psychology, 21(6), 1016-1024.

Gerard, J. M., Buehler, C., Franck, K., \& Anderson, O. (2005). In the eyes of the beholder: Cognitive appraisals as mediators of the association between interparental conflict and youth maladjustment. Journal of Family Psychology, 19(3), 376-384.

Gillham, J. E., Shatté, A. J., \& Reivich, K. (2001). Needed for prevention research: Long-term follow up and the evaluation of mediators, moderators, and lay providers. Prevention $\mathcal{E}$ Treatment, 4(1), 143-156.

Grych, J. H., Seid, M., \& Fincham, F. D. (1992). Assessing marital conflict from the child's perspective: The children's perception of interparental conflict scale. Child Development, 63(3), 558-572.

Harter, S. (1999). The construction of the self: A developmental perspective. New York, NY: Guilford Press.

Harter, S. (2003). The development of self-representations during childhood and adolescence. En M. R. Leary \& J. P. Tangney (Eds.), Handbook of self and identity (pp. 610-642). New York, NY: Guilford Press.

Holmes, J. (2001). The search for the secure base: Attachment theory and psychotherapy. East Sussex: Brunner- Rutledge.

Hoeve, M., Dubas, J. S., Gerris, J. R. M., van der Laan, P. H., \& Smeenk, W. (2011). Maternal and paternal parenting styles: Unique and combined links 
to adolescent and early adult delinquency. Journal of Adolescence, 34(5), 813-827.

Irons, C., \& Gilbert, P. (2005). Evolved mechanisms in adolescent anxiety and depression symptoms: The role of the attachment and social rank Systems. Journal of Adolescence, 28(3), 325-341.

Larson-Hall, J. (2010). A guide to doing statistics in second language research using SPSS. London: Routledge.

MacCallum, R. C., Widaman, K. F., Preacher, K. J., \& Hong, S. (2001). Sample size in factor analyses: The role of model error. Multivariate Behavioral Research, 36(4), 611-637.

Machado, T. S. Fonseca, A. C., \& Queiroz, E. (2008). Vinculação aos pais e problemas de internalização em adolescentes - dados de um estudo longitudinal. International Journal of Development and Educational Psychology, 3(1), 321- 332.

McGorry, P. D., Purcell, R., Hickie, I. B., \& Jorm, A. F. (2007). Investing in youth mental health is a best buy. Medical Journal of Australia, 187, S5-S7.

Merry, S., McDowell, H., Hetrick, S., Bir, J., \& Muller, N. (2004). Psychological and/or educational interventions for the prevention of depression in children and adolescents. The Cochrane Database of Systematic Reviews, 2, 57-69.

Minuchin, S. (1974). Families and family therapy. Cambridge, MA: Harvard University Press.

Mota, C. P., \& Matos, P. M. (2009). Apego, conflito e auto-estima em adolescentes de famílias intactas e divorciadas. Psicologia: Reflexão e Crítica, 22(3), 317-325.

Mota, C. P., \& Matos, P. M. (2010). Interparental conflict and individuation in Portuguese emerging adults: The role of loyalty conflict. Comunicación oral presentada en el 5th Congress of the European Society on Family Relations, Milan, Italia. Disponible en http://www.yagiss.de/en/publications/index.html

Mota, C. P., \& Matos, P. M. (2011a). Adolescência e conflitos parentais: Uma perspectiva de resiliência. En P. M. Matos, C. Duarte \& M. E. Costa (Coords.), Famílias: Questões de desenvolvimento e intervenção (pp. 125-151). Porto: LivPsic.

Mota, C. P., \& Matos, P. M. (2011b). Relationship with parents and individuation process in Portuguese young adults: The role of depression and self-esteem. Comunicación oral presentada en el 5th International
Attachment Conference - IAC - "Attachment: The importance of intimate relationships from the cradle to the grave", Oslo, Noruega.

Mota, C. P., \& Matos, P. M. (2013). Peer attachment, coping and self-esteem in institutionalized adolescents: The mediating role of social skills. European Journal of Psychology of Education, 28(1), 87-100.

Moura, O., Santos, R. A., Rocha, M., \& Matos, P. M. (2010). Children's Perception of Interparental Conflict Scale (CPIC): Factor structure and invariance across adolescents and emerging adults. International Journal of Testing, 10(4), 364-382.

Mruk, C. J. (2006). Self-esteem research, theory, and practice: Toward a positive psychology of self-esteem. New York, NY: Springer Publishing Company Inc.

Ngai, S. S. (2009). The effects of parental care and parental control on the internal assets of adolescent children in Hong Kong. International Journal of Adolescence and Youth, 15(3), 235-255.

Pallant, J. (2011). SPSS -Survival manual (4.a ed.). NSW: Allen \& Unwin.

Parker, J. S., \& Benson, M. J. (2004). Parent-adolescent relations and adolescent functioning: Self-Esteem, substance abuse, and delinquency. Adolescence, 39(155), 519-530.

Preacher, K. J., \& Hayes, A. F. (2004). SPSS and SAS procedures for estimating indirect effects in simple mediation models. Behavior Research Methods, Instruments $\mathscr{G}$ Computers, 36(4), 717-731.

Radloff, L. S. (1991). The use of the center for epidemiologic studies depression scale in adolescents and young adults. Journal of Youth and Adolescence, 20(2), 149-166.

Rocha, M. (2008). O desenvolvimento das relações de vinculação na adolescência: Associações entre contextos relacionais com os pais, pares e par amoroso. Disertación presentada para la obtención del Grado de Doctor, Faculdad de Psicologia y de Ciências da Educación, Universidad do Porto, Oporto, Portugal.

Rocha, M., Mota, C. P., \& Matos, P. M. (2011). Vinculação à mãe e ligação aos pares na adolescência: $O$ papel mediador da auto-estima. Análise Psicológica, 29(2), 185-200.

Rosenberg, M. (1965). Society and the adolescent selfimage. Princeton, NJ: Princeton University Press. 
Rosenberg, M. (1979). Conceiving the self. Malabar, FL: Krieger Publishing Company.

Santos, R. A., \& Matos, P. M. (2007). Implicações da sensibilidade à rejeição e do conflito interparental na vinculação romântica. Psicologia: Teoria, Investigação e Prática, 12(2), 341-359.

Shaw, S. W., \& Dallos, R. (2005). Attachment and adolescence depression: The impact of early attachment experiences. Attachment $\mathcal{E}$ Human Development, 7(4), 409-424.

Southall, D., \& Roberts, J. E. (2002). Attributional style and self-esteem in vulnerability to adolescent depressive symptoms following life stress: A 14-week prospective study. Cognitive Therapy and Research, 26(5), 563-579.

Walper, S., Kruse, J., Noack, P., \& Schwarz, B. (2005). Parental separation and adolescents' felt insecurity with mothers: Effects of financial hardship, interparental conflict, and maternal parenting in east and west Germany. Marriage EF Family Review, 36(3-4), 115-145.
Walper, S., \& Schwarz, B. (2001). Adolescent's individuation in east and West Germany: Effects of family structure, financial hardship, and family processes. American Behavioral Scientist, 44(11), 1937-1954.

Wendt, V., Kroll, S., Beckh, K., Gerhard, K. \& Walper, S. (2002). Dokumentation der Erhebugsinstrumente der 4. Hauptbefragung (Berichte aus der Arbeitsgruppe, "Familien-Entwicklung nach der Trennung" 42/2002). Alemania: Ludwig-Maximilians- Universitat München/Friedrich-Schiller-Universität Jena.

Williams, S. K., \& Kelly, F. D. (2005). Relationships among involvement, attachment, and behavioral problems in adolescence: Examining father's influence. Journal of Early Adolescence, 25(2), 168-196.

Wilkinson, R. B., \& Watford, W. A. (2001). Attachment and personality in the psychological health of adolescents. Personality and Individual Differences, 31(4), 473-484. 
